\title{
Spinodal Decomposition of Chemically Reactive Binary Mixtures
}

\author{
A. Lamorgese and R. Mauri \\ Department of Civil $\&$ Industrial Engineering, \\ University of Pisa, Largo Lazzarino 1, 56122 Pisa, Italy
}

(Dated: March 8, 2016)

\begin{abstract}
We simulate the influence of a reversible isomerization reaction on the phase segregation process occurring after spinodal decomposition of a deeply quenched regular binary mixture, restricting attention to systems wherein material transport occurs solely by diffusion. Our theoretical approach follows a diffuse interface model of partially miscible binary mixtures wherein the coupling between reaction and diffusion is addressed within the frame of nonequilibrium thermodynamics, leading to a linear dependence of the reaction rate on the chemical affinity. Ultimately, the rate for an elementary reaction depends on the local part of the chemical potential difference since reaction is an inherently local phenomenon. Based on 2D simulation results, we express the competition between segregation and reaction as a function of the Damköhler number. For a phase-separating mixture with components having different physical properties, a skewed phase diagram leads, at large times, to a system converging to a single-phase equilibrium state, corresponding to the absolute minimum of the Gibbs free energy. This conclusion continues to hold for the critical phase separation of an ideally perfectly symmetric binary mixture, where the choice of final equilibrium state at large times depends on the initial mean concentration being slightly larger or less than the critical concentration.
\end{abstract}




\section{INTRODUCTION}

The objective of this research is to study numerically the influence of a simple reversible isomerization reaction on the phase segregation of a regular binary mixture, occurring after it is deeply quenched from a single-phase equilibrium state into the (two-phase) unstable range of its phase diagram. Based on results of previous works showing that spinodal decomposition of regular binary mixtures can be convection-driven in low-viscosity systems, ${ }^{1-6}$ herein we further restrict attention to cases in which material transport occurs solely by diffusion, to be able to focus on the competition between segregation and reaction in the absence of any convection-driven coalescence (which is relevant whenever transport can occur by convection as well). In fact, phase separation in a number of reaction-diffusion systems occurs primarily by spinodal decomposition, usually triggered by a thermal or compositional quench. Actually, the necessary displacement from a stable state in the one-phase region into the spinodal range can also occur by reaction, e.g., polymerization of a monomer when the polymer being formed is incompatible with one or more of the other components in the system. Note that in what follows we will not deal with reaction-induced spinodal decomposition in any detail since the focus of this paper is on the competition between segregation and reaction during spinodal decomposition of a binary mixture triggered by a thermal quench. In spite of this, due to its relevance to the design of new materials such as, e.g., monolithic porous polymer or carbon materials, ${ }^{7}$ reaction-induced spinodal decomposition ${ }^{8,9}$ has been studied extensively over the past few decades, mostly by experiment. ${ }^{10-17}$ In fact, the first attempt to investigate instabilities in a binary mixture undergoing phase separation induced by an autocatalytic reaction dates back to Huberman ${ }^{18}$ in 1976 . On the other hand, early experimental observations of the influence of chemical reactions on phase separation include a study of pattern formation in a polymer blend with segregation coupled to a transesterification reaction, ${ }^{19}$ or the introduction of photo-cross-linking reactions to binary and ternary phase-separating mixtures by Tran-Cong and 
collaborators. $^{20-25}$ In fact, a number of works indicated that chemical reactions may slow down the phase separation in polymer blends; in particular, Glotzer et al. ${ }^{26}$ studied by Monte Carlo simulation the phase separation of a binary mixture in the presence of a reversible $\mathrm{A} \rightleftharpoons \mathrm{B}$ reaction, showing that, unlike the nonreacting case, phase separation proceeds only to a certain extent, until the system freezes into a lamellar morphology, due to a suppression of low wavenumber composition fluctuations. ${ }^{27}$ Ever since a number of similar studies have been conducted to further investigate the influence of reversible reactions on binary and ternary systems undergoing phase separation, based on alternative numerical methods and model equations. ${ }^{28-35}$ In particular, Glotzer's results were questioned by Lefever and coworkers ${ }^{36,37}$ as to the thermodynamic coupling between reaction kinetics and diffusion, which cannot be neglected particularly in the vicinity of the critical point. Using nonequilibrium thermodynamics, ${ }^{38,39}$ Carati \& Lefever ${ }^{37}$ presented a more general linear stability analysis showing that there exist four possible cases that include the prediction by Glotzer et al. ${ }^{27}$ regarding a reaction-induced stabilization of low wavenumber composition fluctuations. Herein, we further address reaction and diffusion in a phase-separating regular binary mixture within the frame of nonequilibrium thermodynamics, focusing on the influence of a reversible isomerization reaction as it affects both the linear regime of spinodal decomposition and its late stage, based on a binary fluid diffuse-interface model for regular mixtures with an asymmetric phase diagram. We also address reaction coupled to phase segregation in an ideally perfectly symmetric binary system.

Although many previous works have addressed the competition between segregation and reaction particularly in phase-separating chemically reactive polymer blends, to our knowledge the Damköhler number dependence of segregation statistics for a regular binary mixture undergoing phase separation in the presence of a reversible isomerization reaction has not been discussed in the literature before and constitutes the principal result reported herein. An outline of the remainder of this paper is as follows. The governing equations and numerical methods are briefly outlined in Sect. II. Then, in Sect. III we show the results of numerical simulations of a phase-separating 
chemically reactive binary mixture as a function of the Damköhler number. Conclusions are then presented.

\section{MODEL DESCRIPTION}

We restrict attention to partially miscible, regular binary mixtures of two species $A$ and $B$ with molar fractions $x_{A}$ and $x_{B}=1-x_{A}$, kept at constant temperature $T$ and pressure $P$. In previous works, ${ }^{1,4,40-43}$ such mixtures had been assumed to have components with equal densities, viscosities and molecular weights (i.e., an ideally perfectly symmetric binary system). Herein, we consider mixtures with component species having different physical properties, corresponding to an asymmetric phase diagram. ${ }^{44}$ Now, when the mixture has a uniform composition, the equilibrium state of the system is described by the thermodynamic Gibbs free energy of mixing

$$
\Delta g^{t h}=g^{t h}-\left(g_{A} x_{A}+g_{B} x_{B}\right)
$$

where $g^{\text {th }}$ is the molar free energy of the mixture at equilibrium, while $g_{A}$ and $g_{B}$ denote the molar free energies of the pure species at temperature $T$ and $P$. For regular mixtures, the thermodynamic Gibbs free energy of mixing can be written as the sum of an ideal (or entropic) part,

$$
\Delta g^{i d}=\mathscr{R} T\left[x_{A} \log x_{A}+x_{B} \log x_{B}\right]
$$

( $\mathscr{R}$ being the universal gas constant) which is proportional to the entropy of mixing for an ideal solution, and an enthalpic (so-called excess) part,

$$
\Delta g^{e x}=\mathscr{R} T x_{A} x_{B}\left[\Psi_{A} x_{A}+\Psi_{B} x_{B}\right]
$$


where $\Psi_{i}$ denotes the Margules parameter for the $i$ th species. When the composition of the mixture is nonuniform, following Cahn \& Hilliard, ${ }^{45}$ spatial inhomogeneities in the composition can be accounted for by representing the generalized Gibbs free energy of mixing as the sum of a thermodynamic part and a nonlocal part, i.e.,

$$
\tilde{g}=g^{t h}+\frac{1}{2} \mathscr{R} T a^{2}|\nabla \phi|^{2},
$$

where $a$ is a (coarse-grained) sub-micron-scale length, which represents the typical interface thickness between the two phases at equilibrium. Now, from the expression for the chemical potential for species $i$ for homogeneous solution at constant $T$ and $P$

$$
\mu_{i}^{t h}=\frac{1}{\mathscr{R} T}\left(\frac{\partial c g^{t h}}{\partial c_{i}}\right)_{c_{j \neq i}},
$$

with $c_{i}=c x_{i}$ denoting the mole density of species $i$ and $c=c_{A}+c_{B}$ the total mole density, we see that for a binary system the chemical potential difference $\mu^{t h}=\mu_{A}^{t h}-\mu_{B}^{t h}$ is thermodynamically conjugated to $\phi \equiv x_{A}$, i.e., $\mu^{t h}=\frac{d\left(g^{t h} / \mathscr{R} T\right)}{d \phi}$. With the above definitions we obtain

$$
\mu^{t h}=\mu_{0}+\log \frac{\phi}{1-\phi}+\Psi^{+}(1-2 \phi)-\Psi^{-}\left(1-6 \phi+6 \phi^{2}\right)
$$

where $\mu_{0}=\left(g_{A}-g_{B}\right) / \mathscr{R} T$ while $\Psi^{+}=\left(\Psi_{A}+\Psi_{B}\right) / 2$ and $\Psi^{-}=\left(\Psi_{A}-\Psi_{B}\right) / 2$. This relation was extended to inhomogeneous mixtures by Cahn \& Hilliard ${ }^{45}$ in terms of the generalized (i.e., thermodynamic plus nonlocal) chemical potential difference

$$
\tilde{\mu}=\frac{\delta(\tilde{g} / \mathscr{R} T)}{\delta \phi}=\mu^{t h}-a^{2} \nabla^{2} \phi
$$




\section{A. The governing equations}

We consider a reversible isomerization reaction, $\mathrm{A} \rightleftharpoons \mathrm{B}$. Also, we assume isothermal conditions so that the energy balance is identically satisfied. Now, requiring mass conservation for each species leads to well-known continuity equations for the mole densities $c_{i}(i=A, B)$ :

$$
\begin{aligned}
& \frac{\partial c_{A}}{\partial t}+\nabla \cdot c_{A} \mathbf{v}_{A}=-R \\
& \frac{\partial c_{B}}{\partial t}+\nabla \cdot c_{B} \mathbf{v}_{B}=R .
\end{aligned}
$$

Here, $\mathbf{v}_{A}$ and $\mathbf{v}_{B}$ are the local mean species velocities for $A$ and $B$ while $R$ is the reaction rate (molar consumption rate of $A$ per unit volume). As a rule, the above mass conservation equations must be coupled to the Navier-Stokes equation; however, in what follows we further restrict attention to an isothermal reaction-diffusion system in the absence of flow, i.e., where $c_{A} \mathbf{v}_{A}+c_{B} \mathbf{v}_{B} \equiv 0$. Therefore, Eqs. (8) and (9) reduce to

$$
\frac{\partial \phi}{\partial t}=-\nabla \cdot \mathbf{J}_{\phi}-\tilde{v} R
$$

with $\tilde{v}$ denoting the molar volume $\tilde{v}=c^{-1}=M_{w} / \rho$ (a constant in this case based on our assumption of equal molecular weights). Here, the species balance equation for $A$ has been rewritten in terms of the mole fraction $x_{A} \equiv c_{A} / c \equiv \phi$. Also, the diffusive (or antidiffusive) volumetric flux can be shown to be proportional to the gradient of the generalized chemical potential difference [Eq. (7)] through the relation ${ }^{44,46}$

$$
\mathbf{J}_{\phi}=-D \phi(1-\phi) \nabla \tilde{\mu},
$$


where $D$ is the molecular diffusivity. Note that, based on our derivation of the entropy equation for a binary mixture ${ }^{42}$ the entropy production rate for this reaction-diffusion system is

$$
\frac{\sigma}{\mathscr{R}}=-\frac{1}{\tilde{v}} \mathbf{J}_{\phi} \cdot \nabla \tilde{\mu}-\frac{A}{\mathscr{R} T} R
$$

where the chemical affinity, $A=-\mathscr{R} T \mu^{t h}$, depends solely on the local part of the chemical potential difference. ${ }^{39}$ Now, consistent with our modeling the diffusive volumetric flux as the negative gradient of the chemical potential difference (corresponding to a positive contribution to the entropy production), in this work we are interested in a nonequilibrium thermodynamics approach to reaction rate modeling, ${ }^{38}$ i.e.,

$$
R=-\frac{k}{\tilde{v}} \frac{A}{\mathscr{R} T}
$$

where $k>0$ is an Onsager coefficient which, in fact, can be immediately identified as a reciprocal reaction time (i.e., $k \equiv \tau_{r}^{-1}$ ). Although quite close to chemical equilibrium Eq. (13) is expected to hold to a good approximation, large deviations from a linear flux-force dependence are generally observed for chemical reactions. ${ }^{38}$ In fact, in nonequilibrium thermodynamics of chemical reactions, the rate for an elementary reaction is usually expressed in terms of Arrhenius-type exponentials of the chemical potential (a.k.a. fugacity, or the exponential transform of the chemical potential), so that the relationship between reaction rate and affinity turns out to be nonlinear: $:^{38,47}$

$$
R=-\frac{k}{\tilde{v}}\left(1-e^{-A / \mathscr{R} T}\right)
$$

which obviously reduces to Eq. (13) for $|A| / \mathscr{R} T \ll 1$. Since all results reported herein have been based on Eq. (13), we show in Fig. 1 both reaction rate models [Eqs. (13) and (14)] as a function of composition. From this plot, as the range of concentrations accessed by all of our simulations is 


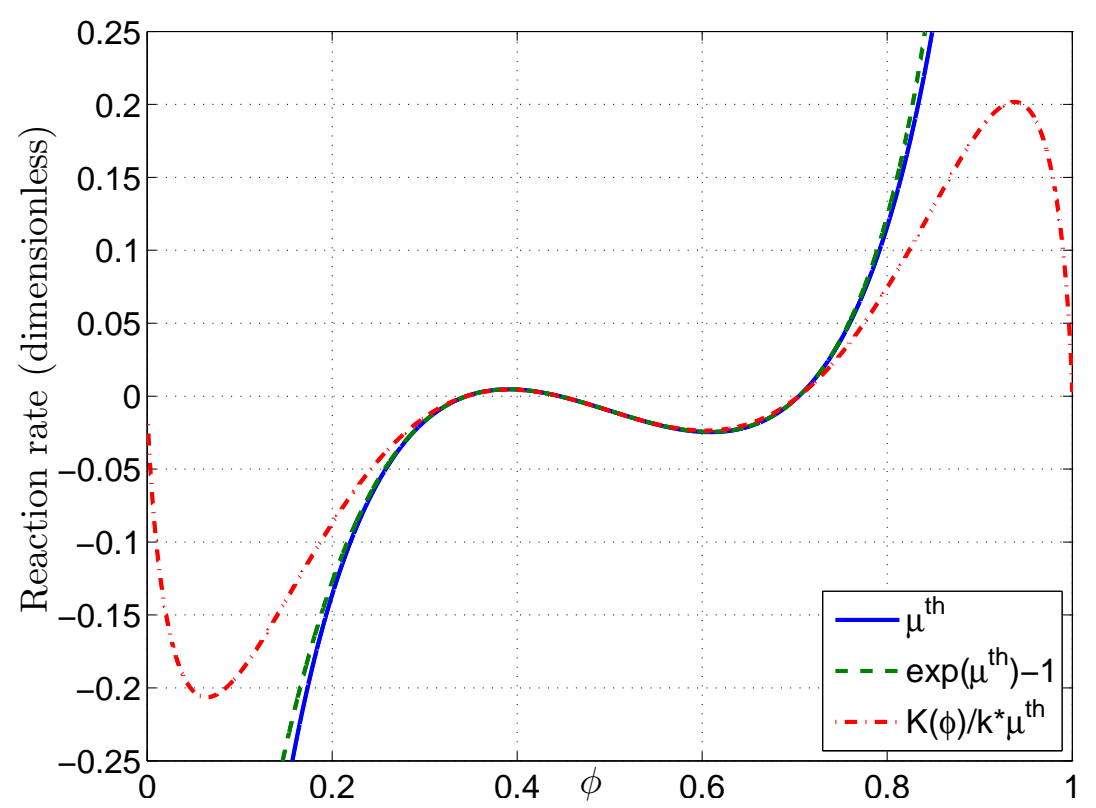

FIG. 1: Dimensionless reaction rates vs. concentration from Eq. (13) (solid), Eq. (14) (dashed), and Eq. (13) with concentration-dependent Onsager coefficient [Eq. (15)] (dot-dashed).

nowhere in the neighborhood of $\phi=0$ or 1 , we can safely conclude that both reaction rate models lead to results that are not qualitatively different. However, it should be acknowledged that both of the above reaction rates show unphysical behavior as they blow up in the dilute limit; in particular, Eq. (13) is divergent at both ends of the composition range (i.e., as $\phi \rightarrow 0$ or 1), while Eq. (14) only diverges as $\phi \rightarrow 1$. In what follows we further restrict attention to Eq. (13). In this case, both divergences of the reaction rate can be eliminated by introducing a concentration-dependent Onsager coefficient of the form:

$$
\frac{\mathcal{K}(\phi)}{k}=\frac{c_{1} \phi(1-\phi)}{1+c_{2} \log \left(\frac{\phi}{1-\phi}\right)} .
$$

As can be seen (Fig. 1), with $c_{1}=4$ and $c_{2}=2 \cdot 10^{-5}$ this model not only provides an excellent approximation to the above reaction rates for the range of compositions of interest in this work, but it also reduces to a linear function of $\phi$ as $\phi \rightarrow 0$ or 1 . 


\section{B. Numerical methods}

The governing equation (in dimensionless form) can be rewritten as follows:

$$
\begin{aligned}
\left(\partial_{t}-\nabla^{2}\right) \phi=-\nabla^{2}\{ & \left.\phi(1-\phi)\left[2 \Psi^{+} \phi+6 \Psi^{-}\left(\phi^{2}-\phi\right)+\left(\frac{a}{L}\right)^{2} \nabla^{2} \phi\right]\right\} \\
& +\nabla \cdot\left\{\left[2 \Psi^{+} \phi+6 \Psi^{-}\left(\phi^{2}-\phi\right)+\left(\frac{a}{L}\right)^{2} \nabla^{2} \phi\right](1-2 \phi) \nabla \phi\right\}-\frac{k L^{2}}{D} \mu^{t h}
\end{aligned}
$$

where lengths and times have been scaled by $L$ and $L^{2} / D$, respectively, with $L$ denoting a macro length scale (which in our case coincides with the periodicity length of the computational domain). Now, since we consider phase-separating mixtures with an asymmetric phase diagram (as it happens when $\mu_{0} \neq 0$, which is the case with the simulation results reported herein), the reaction term in Eq. (16) can be estimated as $O\left(\frac{k L^{2}}{D}\left|\mu_{0}\right|\right)$, while the diffusion term is $O\left(\psi \frac{D\left(\phi_{0}\right)}{D} \frac{L^{2}}{a^{2}}\right)$. Here, $\psi$ is defined as $\psi=-\left.\frac{1}{2} \frac{\partial \mu^{t h}}{\partial \phi}\right|_{\phi_{0}}$, while $D\left(\phi_{0}\right) \equiv D \phi_{0}\left(1-\phi_{0}\right)$. [It turns out that $D\left(\phi_{0}\right) \psi\left(\phi_{0}\right)$ is an estimate of the effective diffusivity that arises in a linear stability analysis (see Appendix).] Therefore, we define the Damköhler number as a reaction-to-diffusion flux ratio:

$$
D a_{L}=\frac{k L^{2}\left|\mu_{0}\right|}{D\left(\phi_{0}\right) \psi\left(\phi_{0}\right)}
$$

Note that, even though $a$ is the physically relevant length scale for transport by molecular diffusion (as reflected in the order-of-magnitude estimate above), our definition incorporates $L^{2} /\left(D\left(\phi_{0}\right) \psi\left(\phi_{0}\right)\right)$ as the time required for transport by diffusion across the whole system size.

We assume periodic boundary conditions, which enables a straightforward pseudospectral spatial discretization. In pseudospectral methods, however, special care must be taken to avoid aliasing errors in the computation of nonlinear terms. Quadratic nonlinearities are usually made alias-free using the so-called padding method, consisting of collocating in physical space on a refined grid with $(3 / 2)^{2}$ as many grid points (in $\left.2 \mathrm{D}\right)$ as the number of active modes in the calculation. Us- 


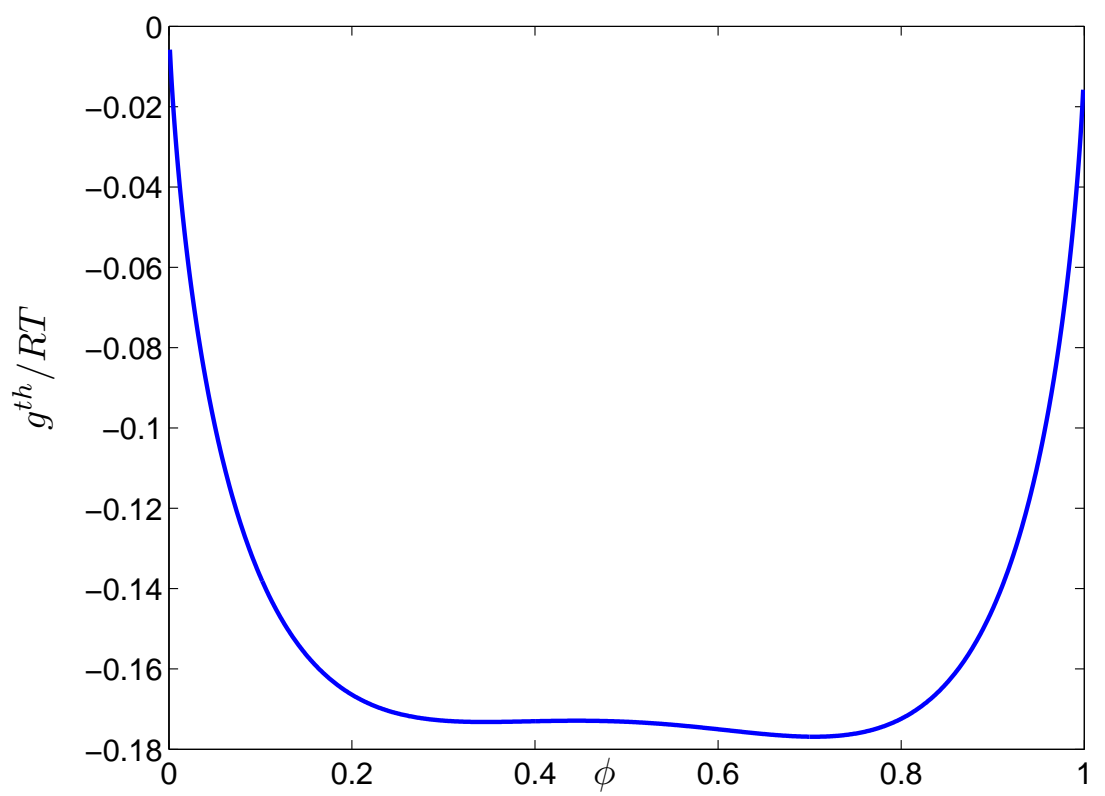

FIG. 2: Thermodynamic Gibbs free energy for a binary mixture with $\Psi^{+}=2.1, \Psi^{-}=0$ and $\mu_{0}=-0.01$. The absolute minimum of the free energy is at $\phi_{e q}^{*}=0.7046$.

ing the padding method, a refined grid with $(5 N / 2)^{2}$ points is required for dealiasing the quartic nonlinearities. ${ }^{48}$ In our production runs, the 3/2-rule has been employed for quadratic, cubic, and quartic nonlinearities, since the remaining aliasing errors were found to be negligible. The Fourier-transformed Eq. (16) can be rewritten as follows:

$$
\frac{d}{d t}\left(e^{k^{2} t} \hat{\phi}_{\mathbf{k}}\right)=e^{k^{2} t} \hat{N}_{\mathbf{k}}
$$

where the Fourier coefficients are denoted by hats, and an integrating factor has been employed for the exact treatment of the purely diffusive term on the LHS of (16). The grid spacing has been chosen so that interface profiles are resolved with three collocation points, i.e., $\Delta x=a / 2$. Equation (18) has been time-integrated using the standard fourth-order Runge-Kutta scheme, ${ }^{1}$ with a variable time step determined by

$$
\Delta t=N_{C} \frac{\Delta x}{V},
$$



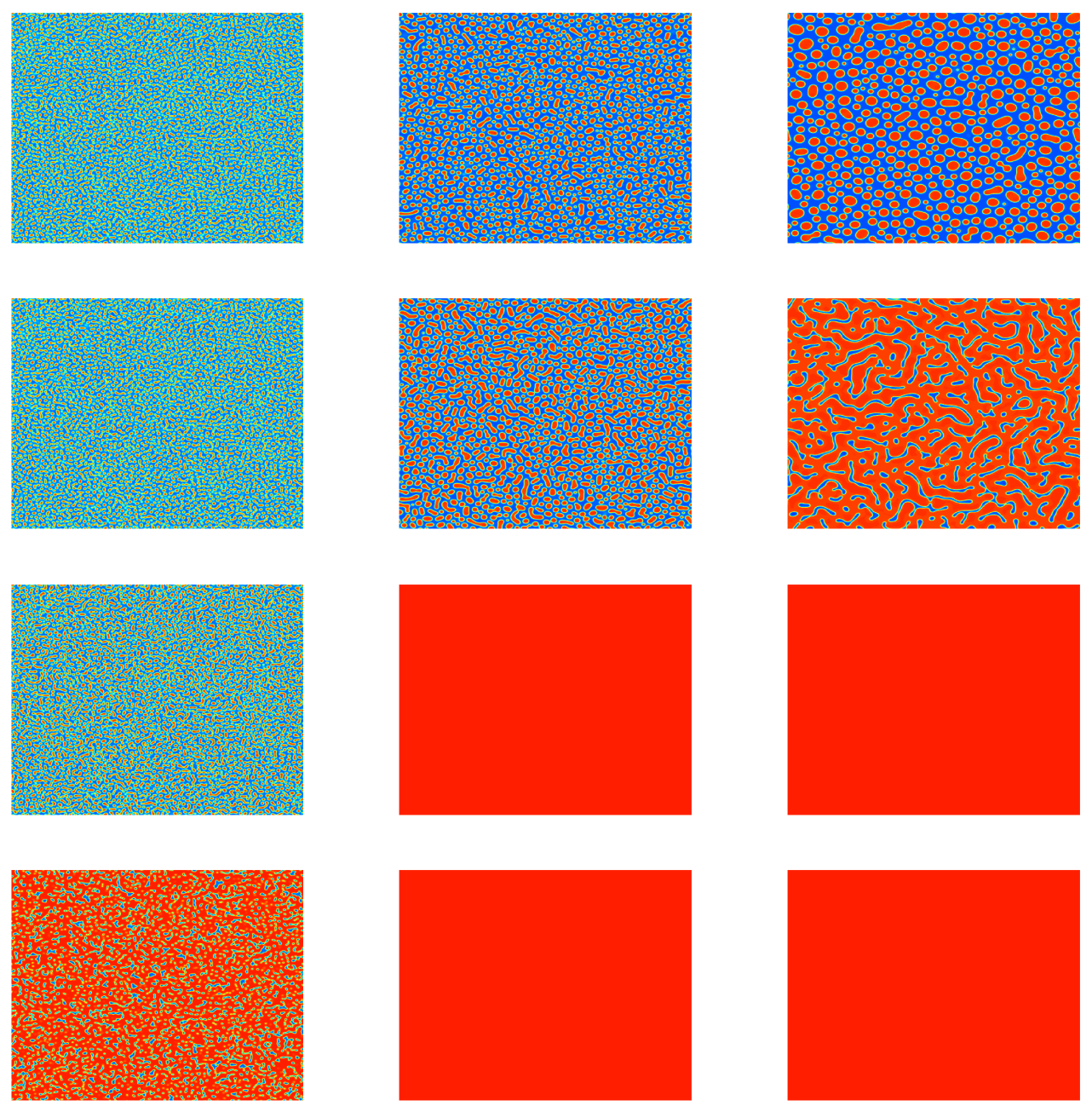

FIG. 3: Snapshots of mass fraction at different (nondimensional) times $t=510^{-2}, 510^{-1}, 2$ (left to right) from phase-field simulations of an asymmetric binary mixture with $\Psi^{+}=2.1, \Psi^{-}=0, \mu_{0}=-0.01$ on a $512^{2}$ grid with Damköhler numbers $D a_{L}=0.531,5.31,53.1,531$ (top to bottom).

where $N_{C}$ is a dimensionless parameter. Also, $\Delta x$ is the dimensionless grid spacing, while $V=$ $\max \left[\left|\phi_{, x}\right|+\left|\phi_{, y}\right|\right]$. This makes our temporal scheme sensitive to the spatial gradients of $\phi$. In addition, $N_{C}$ was chosen such that the time advancement scheme is numerically stable and the smallest dynamical motions are accurately computed. Unfortunately, the non-linearity of the equation prevents a rigorous determination of the stability limit and imposes a trial-and-error determination of the maximum $N_{C}$. In our simulations, we chose $N_{C}=N_{C}^{*}(D a)\left(\right.$ with $N_{C}^{*}(D a)=$ $10^{-3} \div 10^{-2}$ depending on our choice of $\left.D a\right)$, as we found values of $N_{C}>N_{C}^{*}(D a)$ such that the scheme is numerically unstable. 


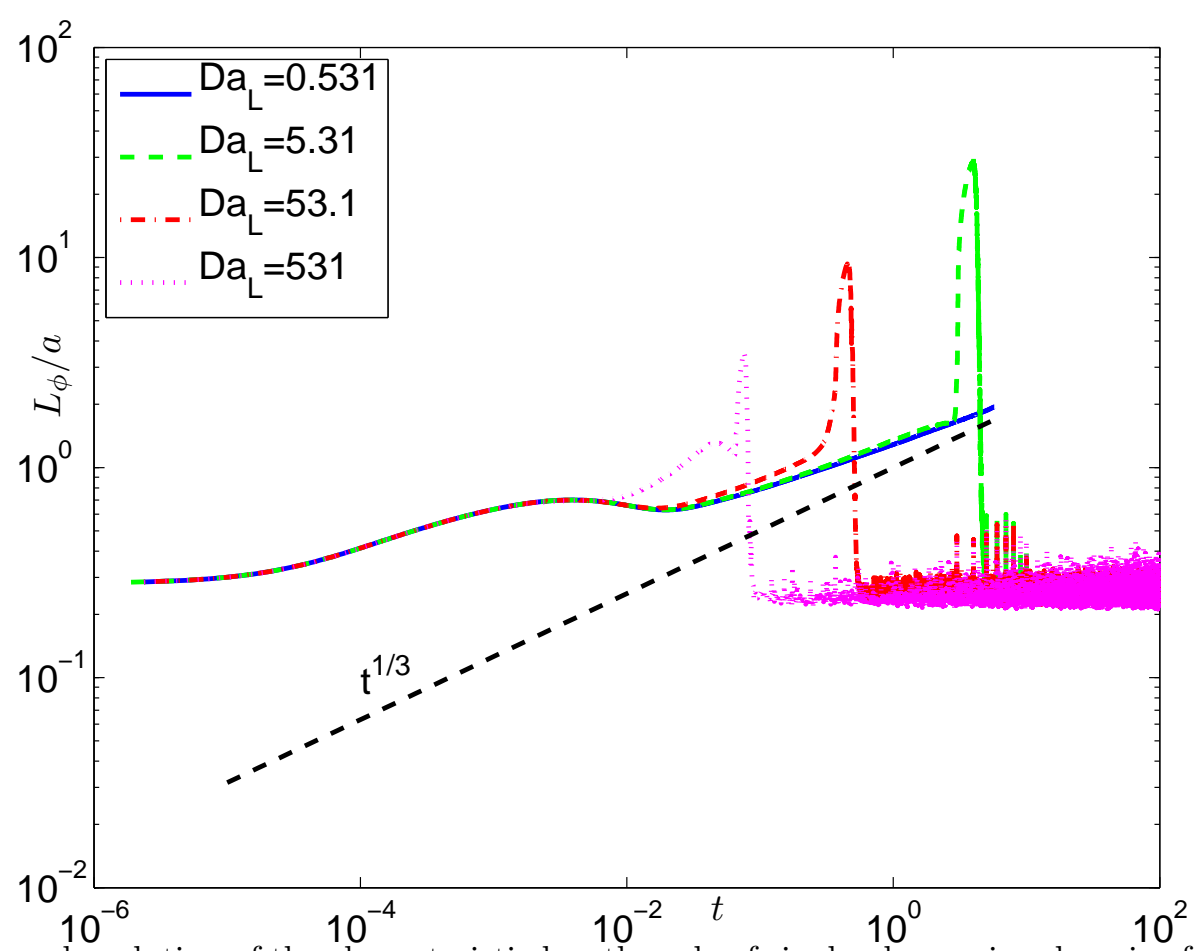

FIG. 4: Temporal evolution of the characteristic length scale of single-phase microdomains from phase-field simulations of an asymmetric binary mixture with $\Psi^{+}=2.1, \Psi^{-}=0, \mu_{0}=-0.01$ on a $512^{2}$ grid and different Damköhler numbers.

\section{RESULTS AND DISCUSSION}

Numerical results from 2D simulations of spinodal decomposition in the presence of the reversible isomerization reaction above are now described. Simulations were run at different Damköhler numbers, $D a_{L}=0.531,5.31,53.1,531$, with $\Psi^{+}=2.1$ and $\Psi^{-}=0$. Using a single Margules parameter, an asymmetric phase diagram was described solely based on the term $\mu_{0}=-0.01$. The corresponding thermodynamic Gibbs free energy is shown in Fig. 2. We took the initial system location between the spinodal points $\phi_{s}^{\beta}=0.39$ and $\phi_{s}^{\alpha}=0.609$, at the relative maximum $\phi_{0}=0.4457$. In particular, at this location the results of a linear stability analysis (see Appendix) hold exactly. Spinodal patterns at different (nondimensional) times and for different values (noted above) of the Damköhler number are shown in Fig. 3. As can be seen, the phase segregation process at $D a_{L}=0.531$ is controlled solely by diffusion, at least for times in the range shown in Fig. 3 . In other words, the characteristic reaction time is so large compared to the characteristic diffusion 


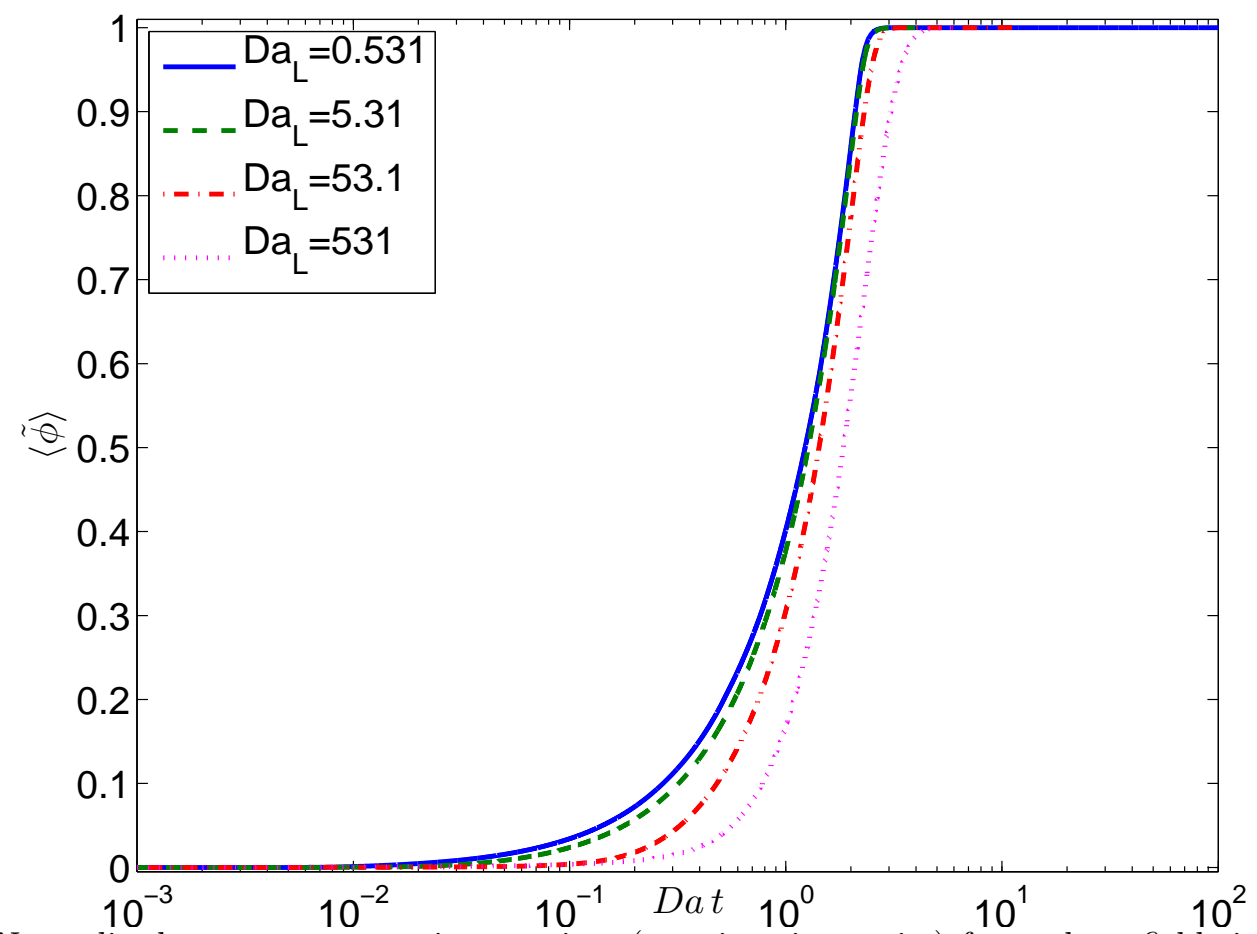

FIG. 5: Normalized mean concentration vs. time (reaction time units) from phase-field simulations of an asymmetric binary mixture with $\Psi^{+}=2.1, \Psi^{-}=0$ and $\mu_{0}=-0.01$ at different Damköhler numbers.

time that diffusion is hardly influenced by reaction in the range of simulated times, leading to the formation of pseudo-spherical nuclei of the minority phase embedded in a continuous phase, which is typical of off-critical spinodal decomposition. Eventually, though, reaction takes over [i.e., for $\left.t>O\left(D a_{L}^{-1}\right)\right]$, driving a phase-separated system towards its single-phase equilibrium state at $\phi_{e q}^{*}=0.7046$. However, at $D a_{L}=5.31$ although reaction is still slow compared to diffusion, at the late stage shown in Fig. 3, after the appearance of the first nuclei, reaction takes over, effectively arresting the phase separation and driving the system towards the absolute minimum for the free energy at $\phi=\phi_{e q}^{*}$. Similar behavior is observed for $D a_{L}=53.1$ and $D a_{L}=531$, though reaction tends to kick in sooner due to a smaller characteristic reaction time as compared to the smaller Damköhler number cases. This competition between reaction and diffusion is also reflected in the temporal evolution of the characteristic length scale of single-phase microdomains (see Fig. 4), 
defined as

$$
L_{\phi}(t)=\frac{1}{\phi_{r m s}^{2}} \sum_{\mathbf{k}} \frac{\left|\hat{\phi}_{\mathbf{k}}\right|^{2}}{|\mathbf{k}|},
$$

with a hat denoting a Fourier transform. As can be seen, after an incubation time, ${ }^{49}$ a diffusive type of scaling is apparent for the characteristic length scale for $D a_{L}=0.531$, i.e., $L_{\phi}(t) \sim t^{\alpha}$, with $\alpha \lesssim 1 / 3$, reaction being too slow to have any effect. However, as previously noted, at larger Damköhler numbers reaction effectively arrests the phase separation, by first accelerating the phase separation in terms of an overshoot in the growth of the characteristic length scale; subsequently, the characteristic length sharply decays to a constant value, corresponding to a single-phase equilibrium state.

Since $\langle\phi\rangle$ is no longer conserved during the segregation process in the presence of reaction, in Fig. 5 we show the temporal evolution of a suitably normalized mean concentration, i.e.,

$$
\langle\tilde{\phi}\rangle=\left\langle\frac{\phi(\mathbf{r})-\phi_{0}}{\phi_{e q}^{*}-\phi_{0}}\right\rangle
$$

where $\phi_{0}$ is the initial composition, while $\phi_{e q}^{*}$ denotes the composition of the final single-phase equilibrium state (with the brackets denoting volume and ensemble averaging). Note that this definition is the same (barring a single-phase final equilibrium state) as the separation depth which had been introduced in previous works ${ }^{1,50,51}$ for measuring the distance of single-phase microdomains from their (two-phase) local equilibrium state. However, as can be seen, even with the smallest Damköhler number the temporal evolution of the separation depth (for this reactive situation) is fundamentally different from that exhibited in the absence of chemical reactions, ${ }^{1}$ wherein after the (delayed) onset of phase separation, local equilibrium is reached well after the appearance of microdomains with sharp interfaces, through a process of composition relaxation that 

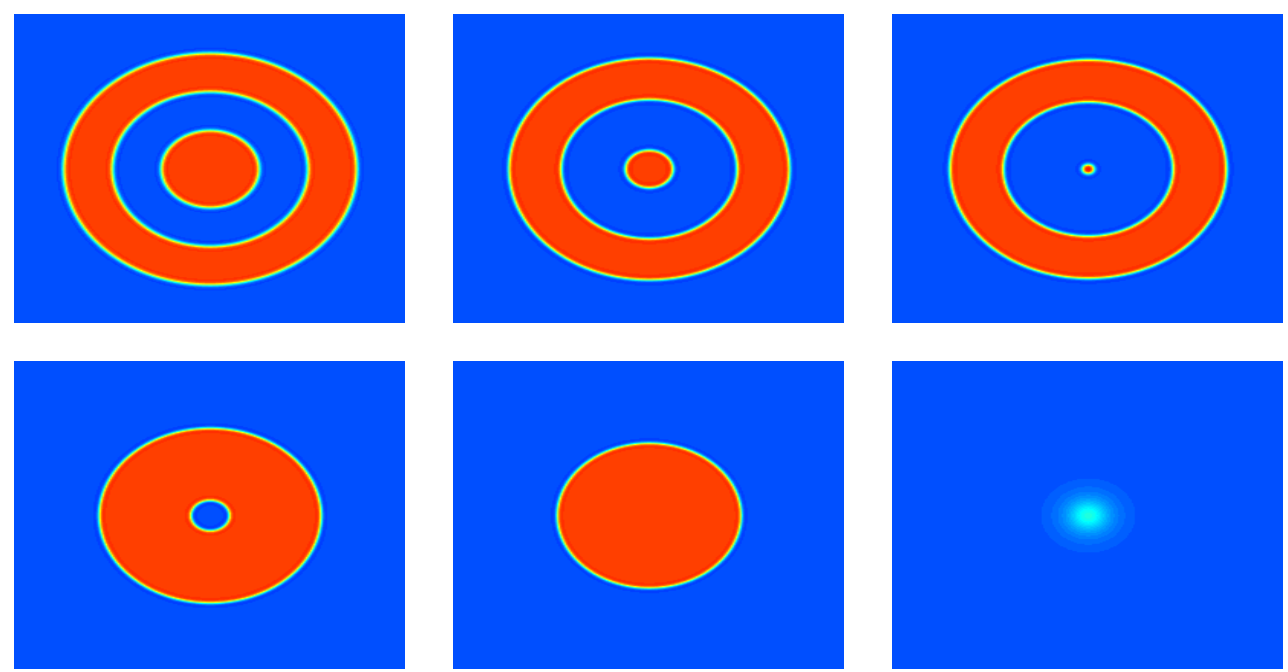

FIG. 6: Snapshots of mass fraction at different (nondimensional) times $t=410^{-3}, 2.4,3.1,12,17$ and 28 (left to right and top to bottom) from phase-field simulations of a perfectly symmetric binary mixture with $\Psi^{+}=2.1, \Psi^{-}=0, \mu_{0}=0$, and $D a=\frac{k a^{2}}{D\left(\phi_{0}\right) \psi\left(\phi_{0}\right)}=4.76$ on a $256^{2}$ grid.

is concomitant to their temporal growth. In the presence of chemical reactions and, in particular, for large Damköhler numbers there is no time for the mixture to phase separate by diffusion as reaction takes over, driving the system to a single-phase equilibrium state corresponding to $\langle\tilde{\phi}\rangle=1$.

Note that the equilibrium points (previously denoted as $\phi_{e q}^{\alpha, \beta}$ ) corresponding to locations on the tangent to the free energy curve in Fig. 2 and representing two coexisting phases are not relevant to the present simulations, since after an initial transient wherein as a result of diffusion two phases might be coexisting for some time, due to the skewness of the phase diagram the system eventually converges to the single-phase (equilibrium) steady state at $\phi=\phi_{e q}^{*}$.

\section{A. Symmetric binary mixture}

The argument above regarding the final equilibrium state for a binary mixture with a skewed phase diagram can be questioned for a perfectly symmetric binary mixture, since in this case it is not obvious that the final equilibrium state should be characterized by a single phase. This question was further investigated by first running simulations with a rectangular strip of the minority $\alpha$ phase embedded in a continuous $\beta$ phase (with both $\phi_{e q}^{\alpha}$ and $\phi_{e q}^{\beta}$ obtained from an equilibrium 
calculation assuming phase coexistence) using $\mu_{0}=0$, the same Margules parameter as above and a large value of the Damköhler number. In these simulations, although the reaction rate is essentially zero within the single-phase microdomains, reactions still occur at the $\alpha / \beta$ interfaces. In fact, with the strip bounded by initially straight interfaces, we saw that nothing happens except for the interfacial thickness tending to become smaller and smaller, since ideally the system would want to minimize the Gibbs free energy by getting rid of the additional energy cost incurred by the presence of interfaces in the computational domain (i.e., by reaching a single-phase homogeneous equilibrium state). Clearly, this cannot happen in this 1D situation. So we ran additional cases with a rectangular strip having corrugated interfaces, such as those obtained using a sinusoidal variation in the $y$ direction to specify the initial interface location. In these cases, we saw that the system tends to get rid of the corrugations, i.e., with a final equilibrium state characterized by straight interfaces. However, for a perfectly symmetric binary system consisting of phase-separated domains initially at equilibrium, interfacial curvature plays a crucial role in driving the system to its final single-phase equilibrium state. This is apparent in simulations with a circular droplet of the minority $\alpha$ phase surrounded by a circular crown of the same phase and embedded in a continuous $\beta$ phase. Again, since reaction is only taking place at the $\alpha / \beta$ interfaces, we saw a cascade of concentric drops collapsing towards the center of the computational domain, with the final equilibrium state consisting solely of a homogeneous $\beta$ phase. As can be seen (Fig. 6), at this value of Damköhler number, it takes a relatively short time for the inner drop to get eroded by reaction and disappear. However, the time it takes for the circular crown to disappear is more than twice as long, since its initial (inner) curvature is more than twice as small as the initial curvature of the inner drop. Next, we looked at an initially phase-separated mixture at equilibrium consisting of a monodisperse array of drops of the minority $\alpha$ phase embedded in a continuous $\beta$ phase. The top panel in Fig. 7 shows a total of 150 drops of the minority phase (having a radius of $8 a$ ) resulting in $\langle\phi\rangle(0)=0.48$; as can be seen, at this value of Damköhler 

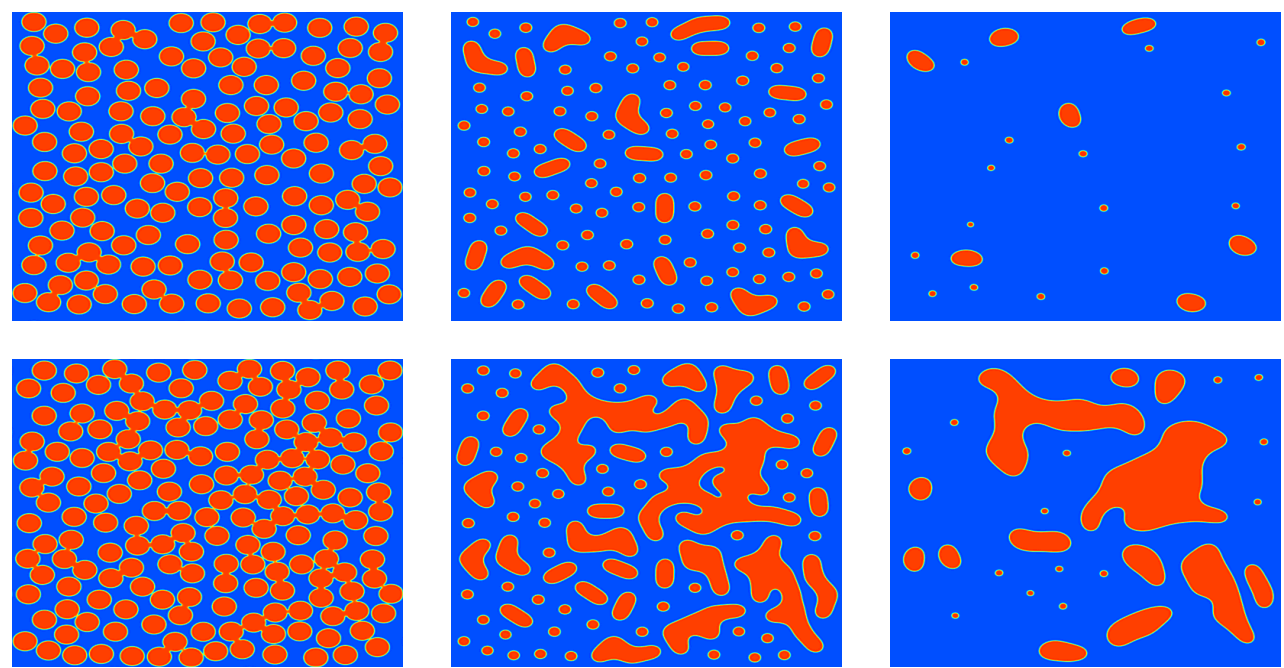

FIG. 7: Snapshots of mass fraction at different (nondimensional) times $t=0.01,1,2.5$ (left to right) from phase-field simulations of a perfectly symmetric binary mixture consisting of a monodisperse array of $\alpha$ phase drops embedded in a continuous $\beta$ phase (at the initial time) with $\Psi^{+}=2.1, \Psi^{-}=0, \mu_{0}=0$, and $D a=\frac{k a^{2}}{D\left(\phi_{0}\right) \psi\left(\phi_{0}\right)}=0.62$ on a $512^{2}$ grid with $\langle\phi\rangle(0)=0.4864$ (top) and $\langle\phi\rangle(0)=0.5150$ (bottom).

number a few drops have time to coalesce by diffusion and only afterwards do such larger domains get eroded by reaction so that the system eventually converges to a homogeneous $\beta$ phase. In fact, this conclusion remains valid even when the total number of drops is increased to 176 , so that the initial mean concentration is $\langle\phi\rangle(0)=0.515>1 / 2$. In this case, too, we saw that the continuous phase wins, i.e., the final equilibrium state consists of a single-phase homogeneous $\beta$ phase. However, for a perfectly symmetric binary mixture at critical concentration, perturbed by delocalized (random) composition fluctuations, with a value of Damköhler number slightly larger than that for the initially monodisperse drops, after the initial diffusion-driven stage of spinodal decomposition, the late stage evolution in the presence of reaction was very sensitive to initial disturbances in the composition that would make the initial mean concentration as either slightly larger or less than the critical concentration, $\langle\phi\rangle=1 / 2$. This bias (which can be controlled using the seed for the random number generator) determines the subsequent evolution towards either the high (with the final equilibrium state being $\alpha$ ) or low end (with the final equilibrium state being $\beta$ ) of the composition range (see Fig. 8). 

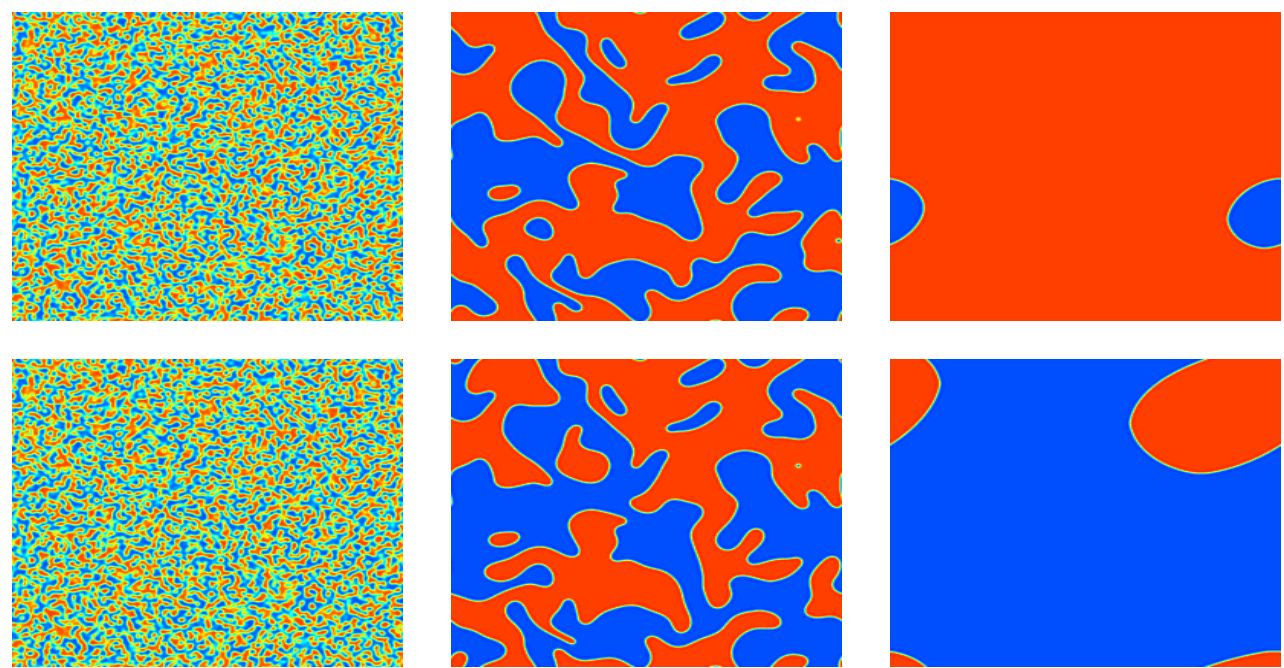

FIG. 8: Snapshots of mass fraction at different (nondimensional) times $t=0.02,1,80$ (left to right) from phase-field simulations of a perfectly symmetric binary mixture at critical concentration (with random noise superimposed at the initial time) with $\Psi^{+}=2.1, \Psi^{-}=0, \mu_{0}=0$, and $D a=\frac{k a^{2}}{D\left(\phi_{0}\right) \psi\left(\phi_{0}\right)}=2.44$ on a $256^{2}$ grid with $\langle\phi\rangle(0)=0.50017$ (top) and $\langle\phi\rangle(0)=0.49994$ (bottom).

\section{CONCLUSIONS}

We have discussed numerical results from 2D simulations of spinodal decomposition of a regular binary mixture with component species having different physical properties (corresponding to an asymmetric phase diagram) in the presence of a reversible isomerization reaction. Our attention has been restricted to systems such as, e.g., polymer melts and alloys, wherein material transport occurs solely by diffusion. Also, the thermodynamic coupling between reaction and diffusion has been addressed within the frame of nonequilibrium thermodynamics, leading to a linear dependence of reaction rate on affinity. We emphasize that, ultimately, the reaction rate should depend solely on the local part of the chemical potential difference, since reaction is an inherently local phenomenon. Using this model, a linear stability analysis for an equilibrium state located in the spinodal range of the phase diagram shows that the effect of reaction is always destabilizing on the linear regime of spinodal decomposition. Furthermore, we discussed the competition between segregation and reaction from spinodal decomposition simulations in 2D at different Damköhler numbers. In particular, we have shown that due to the skewness of the phase diagram for a mix- 
ture with component species having different physical properties, in all cases the system converges at large times to a single-phase equilibrium state, corresponding to the absolute minimum of the Gibbs free energy. This conclusion is still valid for a perfectly symmetric binary mixture; in fact, since there is no skewness in the phase diagram in this case, at steady state the system converges to either the $\alpha$ or $\beta$ phase (obtained from an equilibrium calculation assuming phase coexistence) depending on the initial mean composition being slightly larger or less than the critical concentration, which is ultimately controlled by the seed for the random number generator.

\section{APPENDIX A: LINEAR STABILITY ANALYSIS}

We study the short-time behavior of a regular binary mixture with an asymmetric phase diagram, which is suddenly quenched into the unstable range of its phase diagram at a uniform concentration $\phi=\phi_{0}$ such that $\mu^{\text {th }}\left(\phi_{0}\right)=0$, in the presence of a reversible isomerization reaction. (Note that near-critical conditions are not assumed.) First, we linearize the Cahn-Hilliard equation [Eq. (10)] defining

$$
u \equiv \frac{\phi-\phi_{0}}{\phi_{0}}, \quad \psi=\Psi^{+}-3 \Psi^{-}\left(1-2 \phi_{0}\right)-\frac{1}{2 \phi_{0}\left(1-\phi_{0}\right)}
$$

With $|u| \ll 1$ and $\langle u\rangle(0)=0$, we obtain:

$$
\frac{\partial u}{\partial t}=-2 \psi \nabla^{2} u-\nabla^{4} u+2 \psi D a_{0} u
$$

where the spatial coordinates and time have been made dimensionless based on $a$ and $a^{2} / D\left(\phi_{0}\right)$, respectively, while

$$
D a_{0} \equiv \frac{k a^{2}}{D\left(\phi_{0}\right)}
$$


is a Damköhler number. Equation (A2) follows from a Taylor series expansion for the chemical potential difference around $u=0$ as

$$
\mu^{t h}=-2 \psi \phi_{0} u+\ldots
$$

Using an integrating factor, Eq. (A2) can be rearranged to the form

$$
\frac{\partial u^{\prime}}{\partial t}=-2 \psi \nabla^{2} u^{\prime}-\nabla^{4} u^{\prime}, \quad \text { with } \quad u^{\prime}=u \exp \left(-2 \psi D a_{0} t\right)
$$

which is the same as the linearized Kuramoto-Sivashinsky equation of the nonreactive case. ${ }^{44}$ Now, assuming a periodic perturbation,

$$
u^{\prime}=u_{0}^{\prime} e^{i \mathbf{k} \cdot \mathbf{x}} e^{\sigma^{\prime} t}
$$

we find for the original disturbance

$$
\sigma(k)=k^{2}\left(2 \psi-k^{2}\right)+2 \psi D a_{0} .
$$

Hence we see that

$$
k<\psi\left(1+\sqrt{1+2 D a_{0} / \psi}\right)
$$

corresponds to unstable growth of the disturbance. It follows from Eq. (A7) that the wavenumber which maximizes the exponential growth, $k_{\max }=\sqrt{\psi}$, is the same as for the nonreactive case. However, since $\sigma(0)>0$, the zero mode of the disturbance (i.e., its mean value) is always unstable, in agreement with $\langle\phi\rangle$ not being conserved in the presence of reaction.

Finally, we comment on the large-time behavior of a regular binary mixture with an asymmetric 
phase diagram converging to a single-phase equilibrium state. In this case, too, the Cahn-Hilliard equation can be linearized by first introducing $u$ and $\psi$ as above [Eq. (A1)], with $\phi_{0}=\phi_{e q}^{*}$ denoting the uniform concentration of the final equilibrium state. With $|u| \ll 1$ and $\langle u\rangle(\infty)=0$, we obtain, once again:

$$
\frac{\partial u}{\partial t}=2 \psi^{-} \nabla^{2} u-\nabla^{4} u-2 \psi^{-} D a_{0} u
$$

where $\psi^{-}$denotes the negative part of $\psi$ (note that $\psi^{-}=-\psi$ is positive since $g^{\text {th }}$ is convex in the neighborhood of $\left.\phi_{0}\right)$. Again, this is the same as a linearized Kuramoto-Sivashinski equation. Now, based on our assumption of periodic boundaries, the solution to Eq. (A9) can be written down by inspection:

$$
u(\mathbf{x}, t)=\sum_{\mathbf{k}} \hat{u}_{\mathbf{k}}(0) e^{-\left(2 \psi^{-} k^{2}+k^{4}\right) t} e^{i \mathbf{k} \cdot \mathbf{x}} e^{-2 \psi^{-} D a_{0} t}
$$

Hence, assuming a small value of the Damköhler number, in the final approach to a homogenous equilibrium state inhomogeneities in the composition are smoothed out on a diffusive time given by $a^{2} / D_{e}$, where $D_{e}=2 \psi^{-} D\left(\phi_{0}\right)$ represents an effective diffusivity, and, simultaneously, on a hyperdiffusive time given by $a^{2} / D\left(\phi_{0}\right)$, while $a^{2} D\left(\phi_{0}\right)$ acts as a hyperdiffusivity. Subsequently, reaction kicks in driving the perturbation to zero on a reaction time scale given by $\left(k \psi^{-}\right)^{-1}$.

1 A. G. Lamorgese and R. Mauri, "Phase separation of liquid mixtures," in Nonlinear Dynamics and Control in Process Engineering, edited by G. Continillo, S. Crescitelli and M. Giona (Springer, Rome, 2002), pp. 139-52.

2 R. Mauri, F. Califano, E. Calvi, R. Gupta, and R. Shinnar, "Convection-driven phase segregation of deeply quenched liquid mixtures," J. Chem. Phys. 118(19), 8841-6 (2003). 
3 F. Califano and R. Mauri, "Drop size evolution during the phase separation of liquid mixtures," Ind. Eng. Chem. Res. 43, 349-53 (2004).

4 A. G. Lamorgese and R. Mauri, "Nucleation and spinodal decomposition of liquid mixtures," Phys. Fluids 17, 034107 (2005).

5 P. Poesio, G. Cominardi, A. Lezzi, R. Mauri, and G. P. Beretta, "Effects of quenching rate and viscosity on spinodal decomposition," Phys. Rev. E 74, 011507 (2006).

6 N. Vladimirova, A. Malagoli, and R. Mauri, "Diffusiophoresis of two-dimensional liquid droplets in a phase-separating system," Phys. Rev. E 60, 2037-44 (1999).

7 G. Zhang, G. Liu, Z. Shi, and G. Qiao, "Dynamics of spinodal decomposition coupled with chemical reaction in thermosetting phenol-formaldehyde resin-based solutions and its application in monolithic porous materials," RSC Adv. 4(14), 7068-78 (2014).

8 T. Inoue, "Reaction-induced phase decomposition in polymer blends," Prog. Polym. Sci. 20(1), 119-53 (1995).

9 R. J. J. Williams, B. A. Rozenberg, and J.-P. Pascault, "Reaction-induced phase separation in modified thermosetting polymers," in Adv. Polym. Sci. 128 (Springer, 1997), pp. 95-156.

10 I. A. Zucchi, M. J. Galante, J. Borrajo, and R. J. Williams, "A model system for the thermodynamic analysis of reaction-induced phase separation: Solutions of polystyrene in bifunctional epoxy/amine monomers," Macromol. Chem. Phys. 205(5), 676-83 (2004).

11 P. K. Chan and A. D. Rey, "Polymerization-induced phase separation. 1. Droplet size selection mechanism," Macromolecules 29(27), 8934-41 (1996).

12 P. K. Chan and A. D. Rey, "Polymerization-induced phase separation. 2. Morphological analysis," Macromolecules 30(7), 2135-43 (1997).

13 K.-W. D. Lee, P. K. Chan, and X. Feng, "A computational study of the polymerization-induced phase separation phenomenon in polymer solutions under a temperature gradient," Macromol. Theory Simul. 12(6), 413-24 (2003).

14 J. Oh and A. Rey, "Computational simulation of polymerization-induced phase separation under a temperature gradient," Comput. Theor. Polymer Sci. 11(3), 205-17 (2001).

15 J. M. Stubbs, Y. G. Durant, and D. C. Sundberg, "Polymer phase separation in composite latex particles. 
1. Considerations for the nucleation and growth mechanism," C.R. Chimie 6(11), 1217-1232 (2003).

16 D. Sutton, J. Stanford, and A. Ryan, "Reaction-induced phase-separation in polyoxyethylene/polystyrene blends. II. Structure development," J. Macromol. Sci. B 43(1), 233-51 (2004).

17 E. Girard-Reydet, H. Sautereau, J.-P. Pascault, P. Keates, P. Navard, G. Thollet, and G. Vigier, "Reaction-induced phase separation mechanisms in modified thermosets," Polymer 39(11), 2269-79 (1998).

18 B. Huberman, "Striations in chemical reactions," J. Chem. Phys. 65(5), 2013-9 (1976).

19 H. Tanaka, T. Suzuki, T. Hayashi, and T. Nishi, "New type of pattern formation in polymer mixtures caused by competition between phase separation and chemical reaction," Macromolecules 25(17), 4453$56(1992)$.

20 A. Harada and Q. Tran-Cong, "Experimental verification of a scaling law for phase separation kinetics of reacting polymer mixtures," Macromolecules 29(13), 4801-3 (1996).

21 T. Ohta, O. Urakawa, and Q. Tran-Cong, "Phase separation of binary polymer blends driven by photoisomerization: An example for a wavelength-selection process in polymers," Macromolecules 31(20), $6845-54$ (1998).

22 Q. Tran-Cong and A. Harada, "Reaction-induced ordering phenomena in binary polymer mixtures," Phys. Rev. Lett. 76(7), 1162-5 (1996).

23 K. Kataoka, O. Urakawa, H. Nishioka, and Q. Tran-Cong, "Directional phase separation of binary polymer blends driven by photo-cross-linking with linearly polarized light," Macromolecules 31(25), 8809-16 (1998).

24 Q. Tran-Cong, T. Ohta, and O. Urakawa, "Soft-mode suppression in the phase separation of binary polymer mixtures driven by a reversible chemical reaction," Phys. Rev. E 56(1), R59-62 (1997).

25 Q. Tran-Cong, J. Kawai, Y. Nishikawa, and H. Jinnai, "Phase separation with multiple length scales in polymer mixtures induced by autocatalytic reactions," Phys. Rev. E 60(2), R1150-3 (1999).

26 S. C. Glotzer, D. Stauffer, and N. Jan, "Monte Carlo simulations of phase separation in chemically reactive binary mixtures," Phys. Rev. Lett. 72(26), 4109-13 (1994).

27 S. C. Glotzer, E. A. Di Marzio, and M. Muthukumar, "Reaction-controlled morphology of phaseseparating mixtures," Phys. Rev. Lett. 74(11), 2034-9 (1995). 
J. Verdasca, P. Borckmans, and G. Dewel, "Chemically frozen phase separation in an adsorbed layer," Phys. Rev. E 52(5), R4616-21 (1995).

29 J. J. Christensen, K. Elder, and H. C. Fogedby, "Phase segregation dynamics of a chemically reactive binary mixture," Phys. Rev. E 54(3), R2212-5 (1996).

30 M. Motoyama and T. Ohta, "Morphology of phase-separating binary mixtures with chemical reaction," J. Phys. Soc. Jpn. 66(9), 2715-25 (1997).

31 Y. Huo, H. Zhang, and Y. Yang, "Effects of reversible chemical reaction on morphology and domain growth of phase separating binary mixtures with viscosity difference," Macromol. Theory Simul. 13(3), $280-9(2004)$.

32 R. D. M. Travasso, O. Kuksenok, and A. C. Balazs, "Harnessing light to create defect-free, hierarchically structured polymeric materials," Langmuir 21(24), 10912-5 (2005).

33 P. Dayal, O. Kuksenok, and A. C. Balazs, "Using a single mask to create multiple patterns in threecomponent, photoreactive blends," Langmuir 24(5), 1621-24 (2008).

34 K. Furtado and J. M. Yeomans, "Lattice Boltzmann simulations of phase separation in chemically reactive binary fluids," Phys. Rev. E 73(6), 066124 (2006).

35 H. Li, H. Liu, Z.-Y. Lu, Q. Wang, and C.-C. Sun, "The effect of viscosity on the phase separation dynamics of binary immiscible mixture coupled with reversible reaction," Int. J. Mod. Phys. C 21(12), $1479-88$ (2010).

36 R. Lefever, D. Carati, and N. Hassani, "Comment on Monte Carlo simulations of phase separation in chemically reactive binary mixtures," Phys. Rev. Lett. 75(8), 1674 (1995).

37 D. Carati and R. Lefever, "Chemical freezing of phase separation in immiscible binary mixtures," Phys. Rev. E 56(3), 3127-36 (1997).

38 S. R. de Groot and P. Mazur, Non-Equilibrium Thermodynamics (Dover, New York, 1984).

39 R. Mauri, Non-Equilibrium Thermodynamics in Multiphase Flows (Springer, 2013); ibid., Chap. 7.

40 A. G. Lamorgese and R. Mauri, "Mixing of macroscopically quiescent liquid mixtures," Phys. Fluids 18, 044107 (2006).

41 D. Molin and R. Mauri, "Enhanced heat transport during phase separation of liquid binary mixtures," Phys. Fluids 19, 074102 (2007). 
42 A. G. Lamorgese and R. Mauri, "Liquid mixture convection during phase separation in a temperature gradient," Phys. Fluids 23, 034102 (2011).

43 A. G. Lamorgese, D. Molin, and R. Mauri, "Phase-field approach to multiphase flow modeling," Milan J. Math. 79(2), 597-642 (2011).

44 R. Mauri, R. Shinnar, and G. Triantafyllou, "Spinodal decomposition in binary mixtures," Phys. Rev. E 53, 2613-23 (1996).

45 J. W. Cahn and J. E. Hilliard, "Free energy of a nonuniform system. III. Nucleation in a two-component incompressible fluid," J. Chem. Phys. 31, 688-99 (1959).

46 N. Vladimirova, A. Malagoli, and R. Mauri, "Two-dimensional model of phase segregation in liquid binary mixtures with an initial concentration gradient," Chem. Eng. Sci. 55, 6109-28 (2000).

47 D. Kondepudi and I. Prigogine, Modern Thermodynamics: From Heat Engines to Dissipative Structures (Wiley, 2015), 2nd edn., p. 371.

48 C. Canuto, M. Hussaini, A. Quarteroni, and T. A. Zang, Spectral Methods in Fluid Dynamics (Springer, New York, 1988), p. 84 .

49 A. G. Lamorgese and R. Mauri, "Diffuse-interface modeling of liquid-vapor phase separation in a van der Waals fluid," Phys. Fluids 21, 044107 (2009).

50 N. Vladimirova, A. Malagoli, and R. Mauri, "Diffusion-driven phase separation of deeply quenched mixtures," Phys. Rev. E 58, 7691-9 (1998).

51 N. Vladimirova, A. Malagoli, and R. Mauri, "Two-dimensional model of phase segregation in liquid binary mixtures," Phys. Rev. E 60, 6968-77 (1999). 